\title{
Individualized laparoscopic B-ultrasound-guided microwave ablation for multifocal primary liver cancer
}

\author{
Zhifeng Xu, Zhangwei Yang, Jianghua Pan, Yiren Hu \\ Department of General Surgery, Wenzhou People's Hospital, Wenzhou, China
}

Videosurgery Miniinv 2018; 13 (1): 9-16

DOI: https://doi.org/10.5114/wiitm.2018.72730

\begin{abstract}
Introduction: Liver cancer is one of the most common malignancies of the digestive system. Minimally invasive ablation procedures have become one of the major means for treating unresectable multifocal liver cancer and have been extensively applied in primary and metastatic liver cancer treatment. Laparoscopic B-ultrasound-guided microwave ablation is an example of the progress made in this field.

Aim: To analyze and summarize the results of and experience with laparoscopic B-ultrasound-guided microwave ablation for multifocal primary liver cancer; moreover, the ablation effects were compared between tumors of different sizes.

Material and methods: Laparoscope-guided needle ablation was conducted on 84 lesions from 32 patients with primary liver cancer based on tumor size, quantity, and location. Moreover, the perioperative data, ablation effects according to tumor size, and long-term follow-up results were analyzed.

Results: Among the 84 nodules treated via microwave ablation, tumors measuring $\leq 3 \mathrm{~cm}$ demonstrated complete ablation upon imaging analysis conducted 1 month after surgery. Moreover, 5 of the tumors measuring $>3 \mathrm{~cm}$ demonstrated incomplete ablation. In these cases, a second procedure was performed, until imaging studies confirmed that complete ablation was achieved.

Conclusions: Laparoscopic microwave ablation allows for precise puncture positioning, an effective ablation range, and safe and feasible surgery, which is especially suitable for liver tumors located in sites difficult to access.
\end{abstract}

Key words: liver cancer, laparoscopic microwave ablation, B-ultrasound-guided.

\section{Introduction}

Liver cancer is one of the most common malignancies of the digestive tract, with over 1 million new cases being reported globally every year. Liver cancer is associated with a high malignancy grade, high recurrence rate, high metastasis rate, and poor prognosis. Surgical resection is considered to be the preferred method for treating primary liver cancer, and allows for radical treatment in some patients [1, 2]. However, in the clinical setting, most patients present with multiple lesions located in various hepatic lobes; thus, a low resection rate is a known obstacle in liver cancer treatment. Moreover, some liver cancer patients are insensitive to chemotherapy and radiotherapy and not eligible for surgery; effective treatment for such subsets of patients are currently lacking [2-4]. Over the past few years, minimally invasive ablation technology has been continuously perfected and clinical experience constantly accumulated. Minimally invasive ablation procedures have become one of the major means for treating unresectable multifocal liver cancer and have been extensively applied in primary and metastatic liver cancer treatment. Lapa-

\section{Address for correspondence}

Zhangwei Yang, Department of General Surgery, Wenzhou People’s Hospital, 57 Canghou St, 325000 Wenzhou, China,

phone: +86 15088611336, e-mail: yangzhangwei@126.com 
roscopic B-ultrasound-guided microwave ablation is an example of the progress made in this field.

\section{Aim}

The aim of this study was to explore the application of laparoscopic microwave ablation in treating multifocal primary liver cancer and to analyze the therapeutic effect.

\section{Material and methods \\ Participants}

Herein, data for 32 patients undergoing laparoscopic B-ultrasound-guided microwave ablation in the General Surgery Department of Wenzhou People's Hospital from September 2013 to September 2015 were analyzed. The inclusion criteria were as follows: (1) patients without extra-hepatic metastases from primary liver cancer; (2) patients with $>2$ lesions located in different liver segments, increasing the difficulty of surgical resection; and (3) patients with multiple lesions who were indicated for surgery but demonstrated high surgical risk such as hepatic insufficiency, severe cirrhosis, and portal hypertension. Of the 32 patients, 14 were male and 18 were female, with a median age of $59(48-72)$ years. The patients were informed of the surgical procedure and provided informed consent to participate in this research. All patients first underwent a careful preoperative examination, including definitions of underlying diseases based on the Child-Pugh criteria and tumor morphologic analysis using computed tomography (CT).

\section{Surgical procedure}

Intraoperative laparoscopic ultrasound exploration (Model) was adopted if the precise tumor location was not known (Photo 1). The tumor diameter was measured carefully; echo characteristics as well as the tumor's association with important peripheral structures were recorded. Blood flow distribution and the degree of blood flow in and around the tumor, as well as the vasa vasorum, were observed, to determine the insertion route of the microwave needle. The microwave generator was connected to the cooling circulating system, with the temperature of the condensate water maintained at $3-5^{\circ} \mathrm{C}$. A small incision was made in the abdominal wall above the tumor, and the $14 \mathrm{G}$ microwave needle was inserted into the tumor under laparoscopic guidance. The ab- lation range was set at $0.5-1.0 \mathrm{~cm}$ bordering the tumor lesion; if this could not be achieved, multi-point and multifold cycles were conducted, with each cycle lasting $3 \mathrm{~min}$ at a solidification efficiency of $60 \mathrm{~W}$. The radiation conditions were set at $80 \mathrm{~W}$ and $600 \mathrm{~s}$ if the tumor body diameter was $<3.0 \mathrm{~cm}$ to achieve overall tumor coverage and inactivation. However, 1- or 2-needle microwave ablation was adopted for tumor body diameters of 3-5 cm based on the coagulative necrosis. If the tumor body diameter was $>5 \mathrm{~cm}$, a combination of multi-needle, multi-point, and multi-direction approaches was used, with the needle interval being set at $<1.5 \mathrm{~cm}$ at a duration of 10-15 min. Needle withdrawal was conducted under laparoscopic and ultrasound guidance. The needle was withdrawn while cauterizing the liver, so as to reduce the risks of bleeding due to needle passage and cancer metastasis. After the surgery was completed, bleeding and bile leakage were recorded and treated accordingly.

\section{Postoperative follow-up}

All patients enrolled in this research were followed up by the same surgical team as follows: (1) $\alpha$-Fetoprotein (AFP) and new lesions discovered at the re-examination conducted within 1 month after surgery were deemed as local residue rather than recurrence; (2) follow-up was conducted once every month in the first year, and once every 3 months in the second year; (3) AFP and liver B-ultrasound were the major parameters assessed at the follow-up, but CT and magnetic resonance imaging examinations were carried out if recurrence was suspected; and (4) patients with confirmed recurrence were admitted for treatment again via multimodal therapy according to the type of recurrence.

\section{Statistical analysis}

Continuous data are expressed as mean \pm standard error $(\bar{x} \pm$ SEM), and statistical processing of data was conducted using the $t$-test. The test level was set at $\alpha=0.05$, and values with $p<0.05$ were deemed statistically significant.

\section{Results}

\section{Patient characteristics}

Herein, data for 32 consecutive patients with primary liver cancer who underwent laparoscopic 

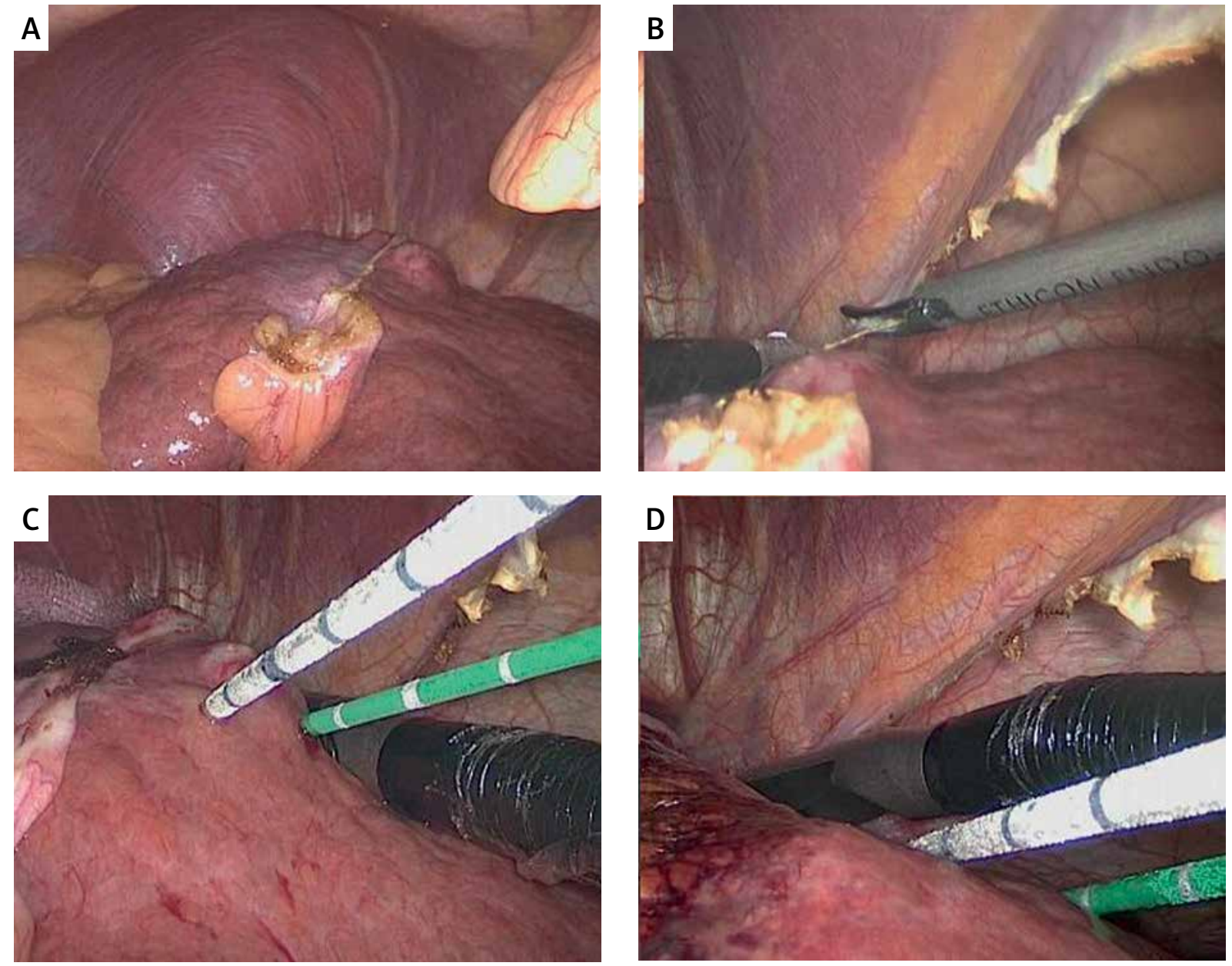

Photo 1. Intraoperative photo showing the procedure of laparoscopic microwave ablation (MWA). Liver cirrhosis and surface mass (A); dissect ligamentum teres hepatis (B); B-ultrasound-guided microwave ablation (C, D)

B-ultrasound-guided microwave ablation from September 2013 to September 2015 were prospectively analyzed. The characteristics of these patients are shown in Table I. The median age of the patients was 59 years, with most cases involving female patients. All patients had viral hepatitis B (100\%); $53 \%$ of patients were classified as having stage $B$ liver function according to the Child-Pugh criteria. A total of 84 nodules in 32 patients were treated via laparoscopic B-ultrasound-guided ablation using a microwave ablation device. Among these cases, 2 additional tumors were discovered intraoperatively.

\section{Short-term data}

The postoperative complications included pain $(n=8)$, fever $(n=6)$, and abdominal distension $(n=6)$. The median postoperative length of stay was
4 days (range: $2-8$ days). Enhanced $\mathrm{CT}$ was carried out 1 month after surgery to determine the therapeutic effects of microwave ablation and tumor residue. The results suggested that all treated necrotic volumes showed a lower density than that observed in the original tumor or even cyst-like low-density necrotic regions; moreover, enhanced CT showed no enhancement within the tumor region (Photo 2). Imaging examination conducted 1 month after surgery showed that a complete ablation rate of $100 \%$ was achieved for nodules with a diameter of $\leq 3.0 \mathrm{~cm}(n=$ $48)$, among the 84 nodular tumors. Among the nodules with a diameter of $>3.0 \mathrm{~cm}(n=36)$, incomplete ablation was achieved for 5 nodules (4 cases); however, complete ablation was confirmed in the second enhanced CT examination conducted 1 month after the second laparoscopic microwave ablation. Recurrence of each lesion and overall in 32 patients at 3 , 
Table I. Demographic data for 32 patients with hepatocellular carcinoma (HCC) undergoing laparoscopic microwave ablation

\begin{tabular}{|c|c|}
\hline Parameter & Value \\
\hline Age [years] & $58.9(48-72)$ \\
\hline Sex (female/male) & $18 / 14$ \\
\hline Abdominal surgical history & $6(18.8 \%)$ \\
\hline Anti-virus treatment & $12(37.5 \%)$ \\
\hline \multicolumn{2}{|l|}{ Child-Pugh grade: } \\
\hline$A$ & $15(46.9 \%)$ \\
\hline B & $17(53.1 \%)$ \\
\hline Tumor number [cm]: & 84 \\
\hline$\leq 3$ & $48(57.1 \%)$ \\
\hline$>3$ & $36(42.9 \%)$ \\
\hline \multicolumn{2}{|l|}{ Tumor size $[\mathrm{cm}]$ : } \\
\hline$\leq 3$ & $2.1 \pm 0.2$ \\
\hline$>3$ & $3.4 \pm 0.1$ \\
\hline \multicolumn{2}{|l|}{ Liver segmental location: } \\
\hline Segment I & $2(2.4 \%)$ \\
\hline Segment II & $11(13.1 \%)$ \\
\hline Segment III & 9 (10.7\%) \\
\hline Segment IV & $8(9.5 \%)$ \\
\hline Segment V & $15(17.9 \%)$ \\
\hline Segment VI & $10(11.9) \%$ \\
\hline Segment VII & $18(21.4 \%)$ \\
\hline Segment VIII & $11(13.1 \%)$ \\
\hline Bile duct or blood vessel proximity & $13(15.5 \%)$ \\
\hline
\end{tabular}

6 , and 12 months after surgery was recorded. We found that the difference in local recurrence after surgery between the two groups was not statistically significant. In terms of overall recurrence and metastasis, 2 cases involved intrahepatic recurrence in other sites; conventional B-ultrasound-guided microwave ablation was indicated owing to the single small lesion and accessible position.

\section{Survival statistics}

Patients were followed up for a median of 16 months (12-20 months). The follow-up results were as follows: (1) 4 (12.5\%) cases involved local tumor recurrence; (2) 5 (15.6\%) involved intra-hepatic metastasis; (3) 4 (12.5\%) involved extra-hepatic metastasis. No cases of death were reported during the follow-up.

\section{Discussion}

Multifocal primary liver cancer is frequently treated via ablation, largely because the tumors are difficult to resect; however, microwave ablation is a common treatment at present [1-4]. Conventionally, microwave ablation for liver cancer is completed under B-ultrasound guidance, but the tumor may be shaded by lung gas or intestinal gas or may be located close to vital regions of the organ, which may affect puncture positioning. Therefore, laparoscopic B-ultrasound-guided microwave ablation has been increasingly applied in clinical settings $[3,4]$. Compared with conventional B-ultrasound-guided microwave ablation, the surgical field is clear and the operation can be conducted safely under direct laparoscopic guidance. However, peri-hepatic tissues and organs can be moved intraoperatively, which may reduce collateral damage to peripheral tissues. The exploratory field of vision is clear and extensive, and is not restricted by abdominal incision size or current imaging technology [5, 6]. Moreover, laparoscopic B-ultrasound-guided microwave ablation can easily identify lesions that are not visible on preoperative imaging examination. In addition, the closest point to the tumor cannot be selected when positioning the puncture point in vitro, which may add to the risk of accessory injury. The radiofrequency ablation needle can be placed more accurately under laparoscopic guidance. In particular, the monitored treatment range and effects are more reliable in cases of multifocal liver cancer requiring multi-needle and multi-operation approaches [5-9]. Thus, laparoscopic B-ultrasound-guided microwave ablation can reduce the chance of intrahepatic bile duct injury, spare excessive coagulation of peripheral non-tumor tissue, and preserve impaired liver function.

Laparoscopic B-ultrasound-guided microwave ablation is superior to the conventional approach in that it allows for ablation in sites difficult to access. In our practice, multifocal liver cancer manifests in different sites; the ablation is carried out from the periphery to the center, as well as from safe to dangerous sites. For tumors near the liver surface that 

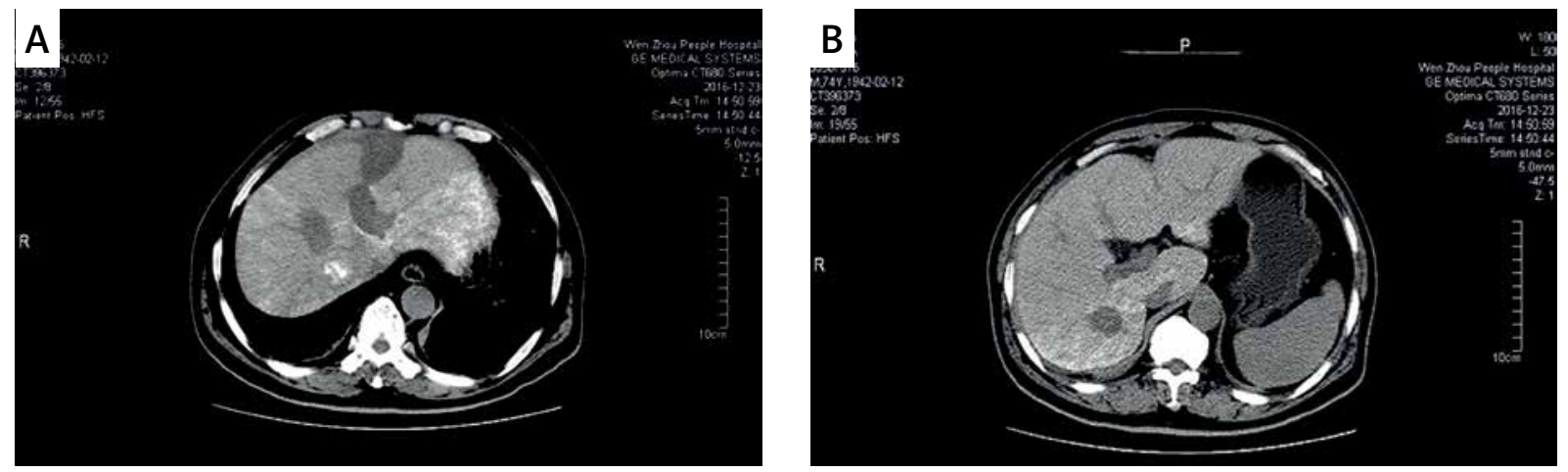

Photo 2. Postoperative contrast-enhanced CT examination (complete ablation)

exhibit hemorrhage after ablation (55 cases in the present study), the ablation time can be delayed or an electric coagulation device can be applied under direct observation. Compared with measures of internal bleeding and digital subtraction angiography (DSA) for cases of hemorrhage after conventional B-ultrasound-guided microwave ablation, direct vision under laparoscopy leads to more accurate hemostatic effects and fewer injuries [10-12]. Furthermore, a tumor exceeding the liver surface is at risk for rupture during ablation. During the procedure, low power $(30 \mathrm{~W})$ ablation is administered for the first $30 \mathrm{~s}$, which is increased to $80 \mathrm{~W}$ thereafter to avoid high temperature-induced tumor rupture. In cases of liver cancer located at the top of the liver near the diaphragm, conventional B-ultrasound-guided operation may injure the diaphragm. In contrast, laparoscopic operation is convenient for observation and positioning. Artificial ascites can be established using saline, such that the liver and diaphragm can be isolated with water while absorbing some of the heat, thus avoiding injury to the diaphragm. In cases of liver cancer located in the lower part of the liver near the gastrointestinal tract, organs close to the tumor can be isolated through traction or compression with pliers under laparoscopic guidance. Thus, injury to adjacent organs such as the gastrointestinal tract can be prevented, and therefore complications such as colonic and gastric perforation can be prevented. It is worth noting that in cases of hepatic tumors located in the left lateral lobe accompanied by cirrhosis, the left hepatic lateral lobe is thin; therefore, the stomach is likely to be damaged during percutaneous microwave ablation. Therefore, during the procedure, the hepatic left lateral lobe can be slightly dissociated under laparoscopic guidance and lifted using the autooperation laparoscopy pole for ablation [12]. In cases of liver tumors located near the great vessel branch and great bile duct branch (33 cases in the present study), poor control over the ablation range may lead to severe complications. All microwave ablation procedures are conducted based on the principle of an inside-out ablation range and the formation of a quasi-circular destruction range behind the needle tip. The preset location of the needle tip can be adjusted as much as possible towards or facing the great vessel branch and great bile duct branch. Thus, the progression of the ablation can be observed, such that the maximal ablation effect is achieved without affecting the great vessel branch or great bile duct branch. Furthermore, previous studies have reported that blood flow will absorb some of the heat from the ablation near the great vessel, which may lead to incomplete ablation. Therefore, hepatic portal occlusion can be induced to arrest the blood flow; however, this method was not attempted in this research $[11,13]$.

In the present study, 32 patients successfully completed the treatment, and no severe complications were reported. The major adverse reactions were pain, fever, and abdominal distention, which improved after the corresponding treatment. However, precautionary measures for severe complications were also taken during the perioperative period. The needle passage transfer rate is reported to be in the range $0.2-12.5 \%$, which is in contrast to that observed in our study $[12,14]$. Needle passage transfer may directly affect the therapeutic effect and therefore warrants attention. In the present study, most tumors were located close to the liver surface or great vessel or were poorly differentiated in terms of pathology. On the basis of our clinical experience, we recommend that needle withdrawal be conducted slowly along with microwave irradia- 
tion (cooling water should be shut down for 10-15 s, followed by shutting down of the microwave needle power supply). This effectively inactivates tumor cells adhering to the needle. Repeated puncture at the same site should be avoided for tumors located close to the liver surface; moreover, needle passage transfer should also be reduced. The incidence of hepatic abscess is estimated at $2-3 \%$. This can be avoided by selecting the optimal approach so as to reduce repeated puncture and heat damage due to ablation [15]. Moreover, antibiotic treatment should be continued for a long time after surgery. All patients in the present study received antibiotics conventionally after surgery. No cases of liver failure were reported in our patients. The literature states that the degree of liver function impairment is significantly worse in patients with a long operation duration than in those with a short operation duration under the same operation conditions. In our study, transient elevation in alanine aminotransferase was observed on the first day after surgery, and returned to the preoperative level 1 week later; no cases of remarkably impaired liver function were observed. Additionally, albumin and total bilirubin levels did not significantly differ before and after treatment. $\alpha$-Fetoprotein levels decreased notably 1 month after treatment compared with that before (Table II). We believe that microwave ablation would cause limited liver function impairment, and the operation duration should be reduced as much as possible under the premise of guaranteeing ablation effects.

Local tumor residual rate is an important indicator of the reliability of microwave ablation or radiofrequency ablation. A previous study showed that complete coverage of the tumor by the coagulation region in $3 \mathrm{D}$ space is the key to determining the effect of microwave ablation. The microwave antenna is placed in the center of the tumor body when the diameter is $\leq 3 \mathrm{~cm}$ so that the treatment can be completed via coagulation on the first attempt. Thus, the survival rate in cases of residual cancer tissue peripheral to the tumor can be reduced; moreover, the coagulative necrosis region can cover $>5 \mathrm{~mm}$ outside the outer edge of the tumor $[15,16]$. Liver tumors with a diameter of $>3 \mathrm{~cm}$ require multipoint and multi-directional punctures for divided treatment. Patients who developed local recurrence in the ablation region or a new lesion in the adjacent liver tissue underwent a second laparoscopic B-ultrasound-guided microwave ablation. The re-examination CT conducted 1 month after surgery did not reveal any tumor residue in cases of tumors with a diameter of $\leq 3 \mathrm{~cm}$; however, in 5 cases (involving 4 patients) among 56 wherein the tumor diameter was $>3 \mathrm{~cm}$, residual lesions were detected. The 5 residual tumors were treated with a second ablation, and no tumor residue was observed on enhanced CT 1 month later. Thus, complete ablation may not be achieved in tumors measuring $>3 \mathrm{~cm}$, because large tumors do not show a regular shape in 3D space, which may lead to omission when designing the ablation range. No obvious local residual lesions are typically identified after the second ablation [17, 18]. Laparoscopic B-ultrasound-guided microwave ablation is a treatment for liver cancer that has the advantages of reproducibility and minimal trauma, adverse reactions, and complications. It is the preferred choice for patients with multiple lesions and with no surgical indications. It can greatly improve the quality of life of patients with advanced tumors and led to the establishment of new conditions for further surgery or chemotherapy for some patients. Tumor size, quantity, site, and adjacent anatomical relationship were found to vary. Therefore, different operation methods were adopted based on different conditions. It is noteworthy that impor-

Table II. Postoperative data comparison

\begin{tabular}{|lcccc|}
\hline Variable & ALT $[\mathrm{U} / \mathrm{I}]$ & TBIL $[\mu \mathrm{mol} / \mathrm{l}]$ & Albumin $[\mathrm{g} / \mathrm{I}]$ & AFP $[\mu \mathrm{g} / \mathrm{I}]$ \\
\hline Before ablation & $67.2 \pm 19.0$ & $34.8 \pm 10.5$ & $39.2 \pm 10.4$ & $451.4 \pm 140.1$ \\
\hline $\begin{array}{l}\text { After ablation } \\
1 \text { day }\end{array}$ & $246.1 \pm 93.0$ & $48.7 \pm 22.1$ & $38.4 \pm 11.3$ & - \\
\hline $\begin{array}{l}\text { After ablation } \\
1 \text { week }\end{array}$ & $98.3 \pm 20.2$ & $40.6 \pm 11.3$ & $37.9 \pm 9.8$ & $401.8 \pm 172.7$ \\
\hline $\begin{array}{l}\text { After ablation } \\
1 \text { month }\end{array}$ & $60.2 \pm 11.2$ & $34.7 \pm 12.1$ & $38.3 \pm 9.7$ & $204.5 \pm 134.4^{*}$ \\
${ }^{*} \leq 0.05$, TBIL - total bilirubin & & & & \\
\hline
\end{tabular}


tance should be attached to the adjacent anatomical characteristics among the numerous factors. Ablation for liver cancer adjacent to other anatomical structures is likely to induce thermal ablation injury and complications or tumor residue owing to an insufficient safety range. Another important factor affecting the effects of ablation is tumor size. A previous study reported that definite ablation can be achieved for tumors with a diameter of $<3 \mathrm{~cm}$. However, tumors measuring $>3 \mathrm{~cm}$ are associated with the possibility of incomplete ablation, which is consistent with the results of this research [16-19]. The minimally invasive and reproducible nature of laparoscopic microwave ablation entails no significant differences in terms of ablation effect and follow-up results between patients receiving a second ablation and those with tumors measuring $<3 \mathrm{~cm}$ in diameter. Therefore, an individualized regimen and strategy for laparoscopic B-ultrasound-guided microwave ablation can guarantee precise positioning and a sufficient therapeutic range for multi-focal primary liver cancer with different sizes in different sites. This contributes to achieving the goal of in situ tumor inactivation.

Objectively, this research is associated with numerous drawbacks, among which the leading problem is that this was a single-center study with a sample size of only 32 patients. The inadequate experience at the beginning of carrying out the research may have also led to differences in the ablation effect. Furthermore, there are numerous defects in the experimental design. To be specific, an excellent comparative study is lacking. Particularly, when certain death case which has different sizes of tumors, the comparison of the effect of microwave ablation will be affected.

\section{Conclusions}

Laparoscopic B-ultrasound-guided microwave ablation is a local treatment regimen for advanced liver cancer, which conforms to the preference for minimally invasive surgery. Compared with conventional B-ultrasound-guided microwave ablation, it allows for determining the degree of cirrhosis under direct vision. Moreover, laparoscopic B-ultrasound-guided microwave ablation can help achieve better ablation effects for tumors located close to the liver surface. More importantly, it can provide a safe ablation environment for multi-focal tumors or those located in sites difficult to access. Further studies are necessary to accumulate additional evidence in favor of this approach

\section{Conflict of interest}

The authors declare no conflict of interest.

\section{References}

1. Sherman M. Epidemiology of hepatocellular carcinoma. Oncology 2010; 78 (Suppl. 1): 7-10.

2. Poon RT, Fan ST, Tsang FH, Wong J. Locoregional therapies for hepatocellular carcinoma: a critical review from the surgeon's perspective. Ann Surg 2002; 235: 466-86.

3. Llovet JM, Bruix J. Novel advancements in the management of hepatocellular carcinoma in 2008. J Hepatol 2008; 48: S20-37.

4. Santambrogio R, Podda M, Zuin M, et al. Safety and efficacy of laparoscopic radiofrequency ablation of hepatocellular carcinoma in patients with liver cirrhosis. Surg Endosc 2003; 17: 1826-32.

5. Liang P, Wang Y, Yu X, Dong B. Malignant liver tumors: treatment with percutaneous microwave ablation: complications among cohort of 1,136 patients. Radiology 2009; 251: 933-40

6. Ong SL, Gravante G, Metcalfe MS, et al. Efficacy and safety of microwave ablation for primary and secondary liver malignancies: a systematic review. Eur J Gastroenterol Hepatol 2009; 21: 599-605.

7. Yamaura T, Sakamoto H, Amikura K, et al. A case of thrombocytopenia after microwave coagulation therapy for multiple metastatic liver tumors. Gan To Kagaku Ryoho 2010; 37: 2579-81.

8. Cucchetti A, Ercolani G, Vivarelli M, et al. Is portal hypertension a contraindication to hepatic resection. Ann Surg 2009; 250: 922-8.

9. Santambrogio R, Podda M, Zuin M, et al. Safety and efficacy of laparoscopic radiofrequency ablation of hepatocellular carcinoma in patients with liver cirrhosis. Surg Endosc 2003; 17: 1826-32.

10. Santambrogio R, Opocher E, Zuin M, et al. Surgical resection versus laparoscopic radiofrequency ablation in patients with hepatocellular carcinoma and Child-Pugh class a liver cirrhosis. Ann Surg Oncol 2009; 16: 3289-98.

11. Hsieh CB, Chang HM, Chen TW, et al. Comparison of transcatheter arterial chemoembolization, laparoscopic radiofrequency ablation, and conservative treatment for decompensated cirrhotic patients with hepatocellular carcinoma. World J Gastroenterol 2004; 10: 505-8.

12. Casaccia M, Andorno E, Nardi I, et al. Laparoscopic US-guided radiofrequency ablation of unresectable hepatocellular carcinoma in liver cirrhosis: feasibility and clinical outcome. J Laparoendosc Adv Surg Tech A 2008; 18: 797-801.

13. Lam VW, Ng KK, Chok KS, et al. Risk factors and prognostic factors of local recurrence after radiofrequency ablation of hepatocellular carcinoma. J Am Coll Surg 2008; 207: 20-9.

14. Minami Y, Kudo M. Radiofrequency ablation of hepatocellular carcinoma: current status. World J Radiol 2010; 28: 417-24.

15. Raoul JL, Sangro B, Forner A, et al. Evolving strategies for the management of intermediate-stage hepatocellular carcinoma: 
available evidence and expert opinion on the use of transarterial chemoembolization. Cancer Treat Rev 2011; 37: 212-20.

16. Llovet JM, Bruix J. Novel advancements in the management of hepatocellular carcinoma in 2008. J Hepatol 2008; 48 (Suppl.): 20-37.

17. Vitale A, Ramirez Morales R, Zanus G, et al. Barcelona Clinic liv er cancer staging and transplant survival benefit for patients with hepatocellular carcinoma: a multicentre, cohort study. Lancet Oncol 2011; 12: 654-62.

18. Pompili M, Saviano A, de Matthaeis N, et al. Longterm effectiveness of resection and radiofrequency ablation for single hepatocellular carcinoma $\leq 3 \mathrm{~cm}$. Results of a multicentre Italian survey. J Hepatol 2013; 59: 89-97.

19. Vavra P, Nowakova J, Ostruszka P, et al. Colorectal cancer liver metastases: laparoscopic and open radiofrequency-assisted surgery. Videosurgery Miniinv 2015; 10: 205-12.

Received: 13.08 .2017 , accepted: 11.10.2017. 